\title{
Biomarkers of neurodegenerative disorders: How good are they?
}

\author{
Varun RACHAKONDA ${ }^{1}$, Tian Hong PAN ${ }^{1}$, Wei Dong LE $E^{2,3,4^{*}}$ \\ ${ }^{1}$ Department of Neurology, Baylor College of Medicine, Houston, TX, USA \\ ${ }^{2}$ Health Science Center, Shanghai Institutes for Biological Sciences/Shanghai 2nd Medical University, Shanghai, China. \\ ${ }^{3}$ Institutes of Biomedical Sciences, Ruijin Hospital, Shanghai $2^{\text {nd }}$ Medical University, 197 Ruijin Er Road, Shanghai, China \\ ${ }^{4}$ Model Organism Division, E-Institutes of Shanghai Universities, Shanghai, China.
}

\begin{abstract}
Biomarkers are very important indicators of normal and abnormal biological processes. Specific changes in pathologies, biochemistries and genetics can give us comprehensive information regarding the nature of any particular disease. A good biomarker should be precise and reliable, distinguishable between normal and interested disease, and differential between different diseases. It is believed that biomarkers have great potential in predicting chances for diseases, aiding in early diagnosis, and setting standards for the development of new remedies to treat diseases. New technologies have enabled scientists to identify biomarkers of several different neurodegenerative diseases. The followings, for instance, are only a few of the many new biomarkers that have been recently identified: the phosphorylated tau protein and aggregated $\beta$-amyloid peptide for Alzheimer's disease (AD), $\alpha$-synuclein contained Lewy bodies and altered dopamine transporter (DAT) imaging for Parkinson's disease (PD), SOD mutations for familial amyotrophic lateral sclerosis (ALS), and CAG repeats resulted from Huntington's gene mutations in Huntington's disease (HD). This article will focus on the most-recent findings of biomarkers belonging to the four mentioned neurodegenerative diseases.
\end{abstract}

Keywords: Alzheimer's disease, Parkinson's disease, Amyotrophic Lateral Sclerosis, Huntington's disease, biomarkers.

\section{INTRODUCTION}

Neurodegenerative diseases are a varied assortment of central nervous system disorders characterized by the progressive loss of neural tissues. These disorders do not have cures because the neurons of the central nervous system cannot regenerate on their own after cell death or damage. Current advances in stem cell research may help in neuroregeneration or neuronal cell replacement as indicated by several new studies [1, 2].

Tremendous efforts have been made in recent years to identify the neuropathological, biochemical, and genetic biomarkers of the diseases so that the diagnosis could be established in earlier stages. Biomarkers are basically biological substances that can be used to indicate the presence or onset of a certain disorder. Although a neuropathologic diagnosis is a currently gold standard, it can usually be done in the form of an autopsy after the patient is dead. Therefore, it will be critical for physicians to

\footnotetext{
*Correspondence:Wei Dong LE

Tel: +86-21-64370045 ext 611209; Fax: +86-21-64370637;

E-mail: wdl@sibs.ac.cn
}

identify disease specific biomarkers at early stages, so that patients have a chance to get an early treatment which may curb the disease progression. Furthermore, the biomarkers should not only be used to help in predicting the onset of the diseases, but also to help in overseeing the rate of progression, or in responding to treatment. For example, for Alzheimer's disease (AD), biomarkers are required to distinguish normal aging from dementia, one disorder from another with dementia, as well as to assist in identifying the exact cause of a dementia. Therefore, the biomarkers for $\mathrm{AD}$, and other neurodegenerative diseases such as Parkinson's disease (PD), amyotrophic lateral sclerosis (ALS), and Huntington's disease (HD), must be reliable and specific, and they should be useful in guiding us to make more accurate diagnosis and better treatment of the diseases.

\section{ALZHEIMER'S DISEASE (AD)}

$A D$ is the most prevalent cause for dementia in the elderly population today. In the United States over four million people are suffering from $\mathrm{AD}$. It is an irreversible, progressive disease characterized by the death of neurons 
and synapses in the brain (mainly in cerebral cortex and hippocampus regions), resulting in the deterioration of cognitive functions such as memory, language, judgment and reasoning, and movement coordination. The majority of the neurons that degenerate in this disease communicate with other neurons using the neurotransmitter acetylcholine. Acetylcholine depletion results in the most striking of symptoms, as the cholinergic system functions in the processes of memory, attention, and learning [3]. Apart from acetylcholine depletion, alterations in acetylcholinesterase (AChE) and butirylcholinesterase (BuChE) can also be observed in AD patients [4]. Combined use of these two markers provides over $90 \%$ sensitivity and specificity in the detection of AD [4].

In 1998, a consensus report listed out the specific criteria and characteristics for the biomarkers of AD [5]. Primarily, biomarkers must be precise, reliable, and inexpensive, while simultaneously detecting a fundamental feature of neuropathology. Secondly, they must be validated in neuropathologically confirmed cases. Also, the markers should be extremely sensitive to any changes in the disease (> 80\% sensitivity). And lastly, the marker specificity should be high enough to distinguish AD from other neurodegenerative disorders. Based on these criteria, scientists have been able to eliminate many substances that do not signify the disease and therefore are of no use to the ultimate goal of obtaining those markers that greatly contribute to improving the diagnosis of AD [6].

The key neuropathological hallmarks consist of the extracellular senile plaques and intracellular neurofibrillary tangles (NFTs) [3]. The major components of the senile amyloid plaques include the infamous $\beta$-amyloid peptides $(\mathrm{A} \beta)$ and the non-A $\beta$ component of AD amyloid (NAC) [7], while the neurofibrillary tangles are fundamentally made up of hyperphosphorylated insoluble forms of the tau protein. The NFTs account for the synaptic degeneration or the atrophy of nerve cells following damage to the axons that they are synaptically connected with. $A \beta$ forms as a result of enzymatic cleavage of the parent Amyloid Precursor
Protein (APP), which is a transmembrane protein encoded by a gene on chromosome 21 . The proteases that are involved in this breakdown of APP are $\alpha-, \beta-$, and $\gamma$ secretases (Fig. 1). A $\beta 42$ aggregates to form oligomers faster than $A \beta 40$ because its 42 -amino acid peptide contains two more hydrophobic amino acid residues. NFTs are composed of paired helical filaments (PHF), which are mainly made up of insoluble hyperphosphorylated tau protein [8]. The biological markers of AD (conveniently summarized in Tab. 1) include both the proposed genetic and biochemical markers which have been carefully studied in recent years.

\section{Genetic markers}

For the most part, scientists are able to link the uncommon early-onset familial AD (FAD) to certain genetic mutations, such as: APP gene mutations and presenilin gene mutations or the late-onset sporadic type to apolipoprotein E (ApoE).

\section{$\beta A P P$ gene mutations}

The $\beta A P P$ gene, the first $\mathrm{AD}$ susceptibility gene to be identified, encodes a transmembrane protein that is glycosylated and its longest isoform contains 770 amino acids. The first genetic mutation linked to AD was found on the $\beta A P P$ gene on chromosome 21 [9]. This finding was confirmed by the fact that patients with trisomy 21 (Down's syndrome) also developed similar plaques and suffered Alzheimer encephalopathy in their later years [10]. $\beta A P P$ is thought to be responsible for a few neuroprotective functions; however, according to the amyloid cascade hypothesis, a mutation on the APP gene will lead to an increase in the expression of the protein, eventually leaving more of the protein to be cleaved and form the harmful $A \beta$ peptides [11].

About twelve missense mutations in the $A P P$ gene have all been found to be related to AD in chronically elevated levels of $A \beta 42$ peptide. Mutational studies of the $\beta A P P$ gene first identified a Glu693Gln missense mutation of the

Tab. 1 Summary of useful genetic and biochemical markers for Alzheimer's disease

\begin{tabular}{cc}
\hline Early-onset, familial type & Late-onset, sporadic type \\
\hline Genetic markers & Genetic markers \\
-Presenilin-1 gene mutations & -ApoE isoforms \\
-Amyloid precursor protein gene mutations & -ApoE polymorphisms \\
-Presenilin-2 gene mutations & \\
Biochemical markers & Biochemical markers \\
-Plasma/CSF A $\beta_{1-42}$ peptide & -CSF A $\beta_{1-42}$ peptide \\
-CSF tau protein & -CSF tau protein \\
-Phospho-tau & \\
\hline
\end{tabular}




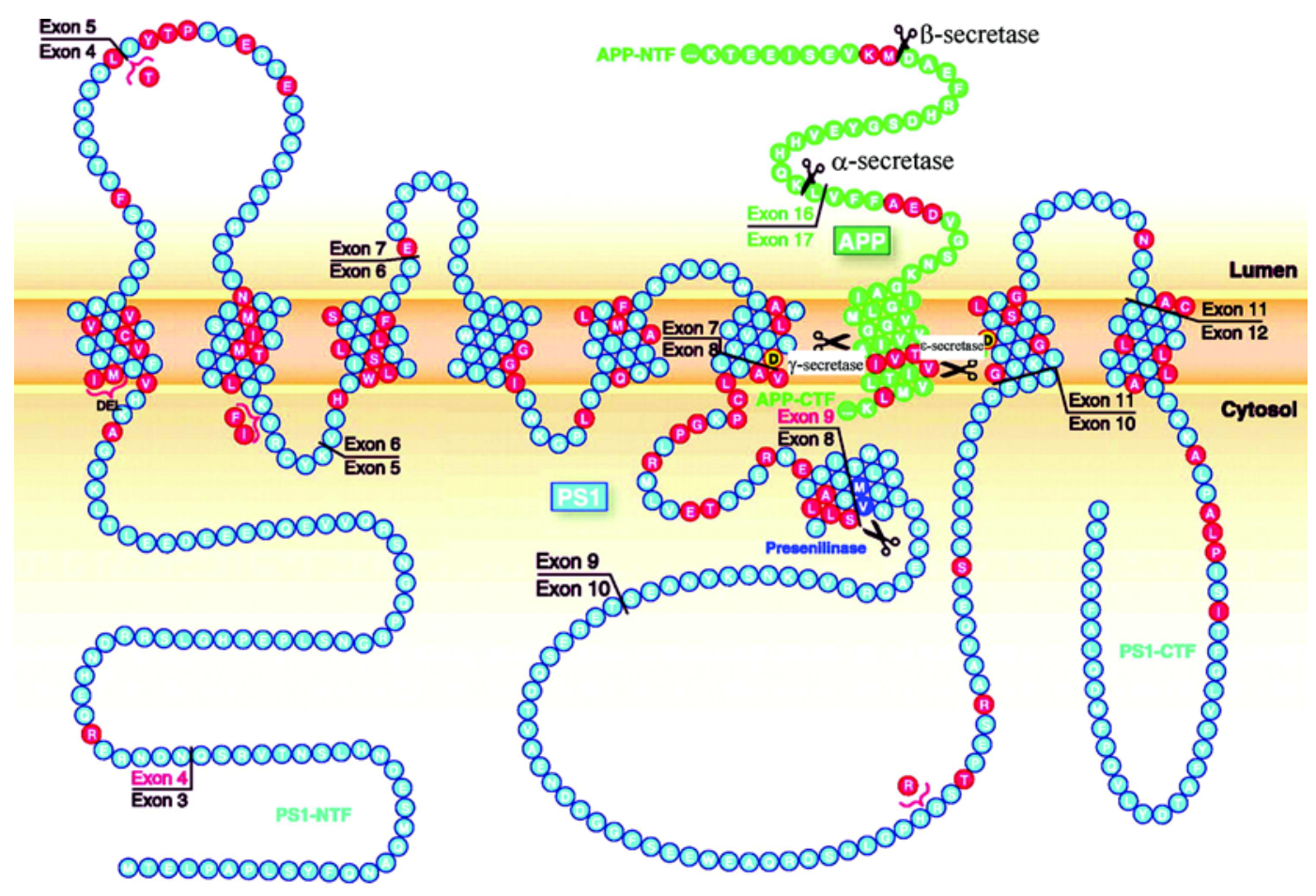

Fig. 1 Shown is the entire amino acid sequence of PS1 (blue circles) and a portion of the COOH-terminal sequence of APP (green circles). Mutations in each molecule known to cause familial AD are depicted in red. The principal sites at which the $\beta-, \alpha-, \gamma-$, and e-protease cleavages of APP occur are indicated by small scissors. Replicated with permission.

$\beta A P P$ gene in affected and at-risk members of families with hereditary cerebral hemorrhage with amyloidosis of the Dutch type [12]. Certain mutations have been hypothesized to influence the processing of $\beta A P P$. Mutations at codons 716 and 717 lead to a selective increase in the production of $A \beta$ peptides ending at residue 42/43 [13]. The Lyc670Asn/Met671Leu mutation, on the other hand, appears to augment the production of both $A \beta 40$ and $A \beta 42 /$ 43 [14], whereas the Ala692Gly mutation exerts a more complicated effect on $\beta A P P$ process causing impaired $\alpha$ secretase cleavage, increased heterogeneity of secreted $A \beta$ species, and increased the hydrophobicity of the $A \beta$. The Ala692Gly mutation also has clinical features in some cases similar to those of hereditary cerebral hemorrhage with amyloidosis-Dutch type, and in other cases more similar to AD but with somewhat subtle differences in the size of the amyloid cores. The Glu693Gln mutation causes an increased propensity for $A \beta$ to form fibrils [15].

\section{Presenilin gene mutations}

Mutations on the two homologous presenilin genes: presenilin 1 (PS1) - located on chromosome 14, and presenilin 2 (PS2) - located on chromosome 1, are responsible for over half of the known familial AD cases. As illustrated in Fig. 1 [11], the presenilin genes code for proteins known as presenilins, which initiate and control the APP proteolysis into smaller peptides [16]. A missense mutation on one of these genes would cause an abnormal increase in the overall proteolytic activity of the APP, thus producing more $A \beta$ peptides or more specifically $A \beta 42$ [17].

To date, more than 120 different mutations have been discovered in the PS1 gene. The majority of these mutations are missense mutations giving rise to the substitution of a single amino acid. These mutations are predominantly located at highly conserved transmembrane domains, at/ near putative membrane interfaces, or in the $\mathrm{N}$-terminal hydrophobic or C-terminal hydrophobic residues of the putative TM6-TM7 loop domain. Two splicing defect mutations have been identified. One involves a point mutation in the splice acceptor site at the 59-end of exon 10 (in some exon numbering systems, exon 10 is labeled as exon 9) [18], and the other arises from the deletion of a $\mathrm{G}$ nucleotide from the splice donor site at the 39-end of exon 5 [19]. The wide scattering of missense mutations leads to the speculation that most of FAD-related mutations end up with specific functional alteration.

Mutational analyses uncovered eight different missense 
mutations in the PS2 gene in families segregating early onset forms of AD. For the first mutation (Asn141Ile), which was detected in a proportion of families of Volga German ancestry, the FAD locus was independently mapped by genetic linkage studies to chromosome 1 . The second (Met239Val) was discovered in an Italian pedigree [20]. Compared with mutations in PS1, screening of large data sets reveals that PS2 mutations are likely to be less frequent [21]. However, PS1 and PS2 seem to have similar or overlapping functions. This hypothesis was supported by the fact that PS2 mutations, like PS1 mutations, could increase the secretion of long-tailed A $\beta$ peptides [22].

\section{ApoE polymorphisms}

The Apolipoprotein (ApoE) itself is a plasma protein that is involved in the transport of cholesterol. In the central nervous system, ApoE is implicated in the growth and repair of the nervous system during development or after injury [23]. ApoE gene is located on chromosome 19 and presents as 3 alleles: $\varepsilon 2, \varepsilon 3, \varepsilon 4$. The $\varepsilon 4$ allele of the ApoE is associated with the early-onset familial and late-onset sporadic forms of AD. It has been hypothesized that the $\varepsilon 4$ allele is involved in enhanced aggregation and/or decreased clearance of $A \beta$. A $\varepsilon 4$ allele was detected in about $40-50 \%$ of all AD patients [24]. However, based on the criteria for biomarkers, given the high sensitivity (93\%) and low specificity (55\%), ApoE alone could not serve as an absolute diagnostic marker [25]. Therefore, ApoE is regarded as a risk factor indicator rather than an actual genetic marker of $\mathrm{AD}$.

\section{Biochemical markers}

The most promising biochemical markers of AD have been found through the analysis of cerebrospinal fluid (CSF). For instance, in all forms of AD, CSF tau levels were found to be increased [26]. In addition to CSF markers, positron emission tomography (PET) imaging with the $\left[{ }^{18} \mathrm{~F}\right] \mathrm{FDDNP}-\mathrm{PET}$ appears to be very promising for $\mathrm{AD}[27,28]$.

\section{CSF $A \beta 42$}

As stated earlier, $A \beta$ is processed through the proteolytic activity of the APP. A $\beta 42$ along with $A \beta 40$ is secreted into the extracellular space and biological fluids, including CSF. However a decrease in the concentrations of CSF-A $\beta 42$ has been observed in many patients, thus making CSF-A $\beta 42$ a considerable indicator of AD [26]. The sensitivity of CSF$\mathrm{A} \beta 42$ to $\mathrm{AD}$ is around $80-90 \%$, allowing it to distinguish $\mathrm{AD}$ from normal aging and depression. It is known that CSF-A $\beta 42$ also increases in some patients with frontotemporal dementia, dementia with Lewy bodies, vascular dementia, and Creutzfeldt-Jakob disease; but in AD, there exists a decrease in levels of $A \beta$ in CSF, which reflects cerebral amyloid deposition. The reason for that may lie in that $A \beta$ peptide from the CSF aggregates to form plaques in the brain as $\mathrm{AD}$ progresses, thereby, lowering its concentration in the CSF. CSF-A $\beta 42$ cannot be solely used as a biomarker for $\mathrm{AD}$, but when used in combination with other AD biomakers, the sensitivity and specificity increase and it appears to be remarkable for diagnosis.

\section{CSF total tau}

Many studies showed that the total levels of the tau protein in the cerebrospinal fluid (CSF-tau) were found to have dramatically increased in AD patients [26]. The tau protein have been proved to be responsible for binding to microtubules in neuronal axons, thus promoting the microtubule stability and assemblage. However, so far it remains unclear as to why this abnormal increase in CSFtau occurs. Although the CSF-tau seems to provide a very high sensitivity for AD, it lacks specificity against other dementias [29].

\section{Combination of CSF-A $\beta 42$ and CSF-tau markers}

As opposed to being used alone, scientists have found that the combination use of the CSF biomarkers may greatly improve their effectiveness - increasing both their sensitivity and specificity for picking out AD patients. In certain studies, the sensitivity of the combination went up to as much as a little over $90 \%$ [30]. The specificity of the combined test was about $86 \%$, well meeting the criteria set for reliable biomarkers of AD. For example: The specificity is $55 \%$ for $A \beta 42$ alone or $65 \%$ for tau alone, indicating that the combination of the two should be the ideal biochemical markers set for $\mathrm{AD}$ [31]. In fact, the combination of several CSF biomarkers in conjunction with other diagnostic methods could be generally used to ensure high accuracy of the clinical diagnosis.

\section{CSF Phospho-tau (P-tau)}

The human tau contains many hosphorylation sites. Tau hyperphosphorylation leads to tau aggregation which subsequently results in the formation of NFTs. The ability of P-tau to discriminate between AD and normal aging shows a mean sensitivity of $70 \%$ and specificity of $94 \%$, while the mean level of increase in AD compared with controls is approximately 250\% [32]. Data on the specificity of CSFphospho-tau is sparse. However, it is believed that Ptau may be a big help in discriminating between AD and these dementias. Further, it has been noted that after acute stroke, there was a marked increase in CSF total tau, while CSF phospho-tau did not change [33], indicating rather than a marker for neuronal damage, as CSF total tau, CSF phospho-tau specifically reflects the phosphorylated tau, 
and in turn the formation of NFTs.

\section{p97/Melanotransferrin (Mtf)}

The expression of p97 or melanotransferrin (Mtf) was initially detected at high levels as a marker of malignant melanoma cells. Later on it came to be known that due to its high sequence homology with human lactoferrin and serum transferrin, Mtf belongs to the group of iron binding proteins. Studies showed that Mtf content in serum was elevated in AD patients, which lead to the potential use of Mtf as an biochemical marker [34, 35]. However, one study showed that when analyzed by SDS-PAGE under non-reducing conditions, Mtf contents in serum were not significantly altered between controls and patients with mild or moderate stages of AD [36]. Further studies should be carried out to confirm that claim.

\section{PET Molecular imaging of $A D$}

2-(1-(6-[(2-[18F]fluoroethyl)(methyl)amino]-2naphthyl)ethylidene)malononitrile ( $\left[{ }^{18} \mathrm{~F}\right]$ FDDNP)-PET has been found to be able to cross the Blood-Brain-Barrier (BBB) due to its high lipophilicity, permitting it to determin the locolization and load of neurofibrillary tangles and senile amyloid plaques in vivo in the human brain. The discovery of a new binding site to $A \beta 40$ fibrils as a result of FDDNP binding also opens a new opportunity for early treatment of $\mathrm{AD}$. PET imaging in vivo may improve current clinical approaches with accuracy rates of 70-90\%. The combined use of the $\left[{ }^{18} \mathrm{~F}\right] \mathrm{FDDNP}-\mathrm{PET}$ labeling system and other diagnostic tests give support to early diagnosis of AD [28]. A recent study using this method examined the in vivo characteristics of $\left[{ }^{18} \mathrm{~F}\right] \mathrm{FDDNP}-\mathrm{PET}$ in patients with $\mathrm{AD}$ and found the relative residence time of the probe in brain regions affected by AD was significantly greater than in control groups [37]. Indeed, $\left[{ }^{18} \mathrm{~F}\right]$ FDDNP-PET scanning in $\mathrm{AD}$ patients show sensitivity at early stages of the disease, before clinical evidence of cognitive decline [27]. $\left[{ }^{18} \mathrm{~F}\right]$ FDDNP-PET imaging of at-risk populations early and throughout the progression of the disease could help in elucidating the etiology of AD by correlating neuropathology with functional loss. Early and longitudinal detection of SPs and NFTs will allow for expedient clinical trials on patients early in the development of AD-a population that would benefit most from promising treatments geared toward reversing the effects of AD. However, an understanding of the molecular requirements of FDDNP binding may help in the optimization of the A $\beta$ anti-aggregation potency of experimental drugs [27].

\section{PARKINSON'S DISEASE (PD)}

$\mathrm{PD}$ is a neurodegenerative disorder, which affects a little over one percent of all people over the age of 55. It is pathologically hallmarked by the degeneration of a neural connection, more specifically dopaminergic neurons between the substantia nigra (SN) and the striatum. Scientists determined that a great majority of dopamineproducing cells in the substantia nigra are lost in patients with PD. As these neurons are destroyed, the clinical signs which characterize PD such as the slowed movements, rigidity and tremors start to appear.

Another key neuropathological mark of PD is the formation of Lewy bodies, which are cytoplasmic inclusions primarily composed of the $\alpha$-synuclein protein. Lewy bodies are present in the dopaminergic neurons of the $\mathrm{SN}$ and other brain regions like the cortex and magnocellular basal forebrain nuclei.

\section{Genetic markers}

In a small number of families, PD is inherited in a Mendelian autosomal dominant or autosomal recessive fashion. Genetic linkage and positional cloning studies in some of these families have identified several causative single gene mutations (namely six genes: $\alpha$-synuclein, Parkin, $U C H$ L1, PINK1, DJ-1 and NR4A2). Furthermore, four loci (PARK3, PARK8, PARK9, PARK10) across the human genome conceal unknown genes (as can be seen in Tab. 2) $[38,39]$. These loci can only be characterized by the phenotypes they produce. These findings lead to intense research efforts to discover how these mutations cause neurodegeneration. The most important of these genes have been discussed as below.

\section{$\alpha$-synuclein gene}

Mutations on the $\alpha$-synuclein gene, which is located on chromosome 4, are a characteristic of Parkinson's disease and they occur in most forms including the rare earlyonset familial form of PD. Polymeropoulos and his colleagues first reported linkage of autosomal dominant PD to chromosome 4q21-23 in an Italian-American family (Contursi family) with typical clinical and pathological features of PD [40]. Further analysis of this region identified a mutation of G to A (G209A) in the $\alpha$-synuclein gene that plays an important role in dopamine associated oxidative damage. Several lines of evidence suggest a toxic mechanism caused by the aggregated protein. However, this mutation was found to be a very rare case of familial PD, having been found in only three other families with autosomal dominant PD. Another mutation of the $\alpha$ synuclein gene was identified in a German family in which there was a single base-pair change at position 88 from $\mathrm{C}$ to $\mathrm{G}(\mathrm{C} 88 \mathrm{G})$ which resulted in an alanine to proline substitution at position 30 of the $\alpha$-synuclein protein [41]. To date, this mutation has not been detected in any other family. 
Tab. 2 Genes for familial Parkinson’s disease

\begin{tabular}{|c|c|c|c|c|c|}
\hline Gene/locus & Location & Inheritane & Onset & Distinctive clinical features & Lewy bodies \\
\hline parkin $=P A R K 2$ & $6 q 25$ & AR & Early-juvenile & $\begin{array}{l}\text { Frequent dyskinesia/dystonia } \\
\text { Slow progression }\end{array}$ & No* \\
\hline$D J-1=P R R K 7$ & 1p36 & AR & Early & $\begin{array}{l}\text { Focal dystonia } \\
\text { Slow progression } \\
\text { Psychiatric symptoms }\end{array}$ & - \\
\hline$P I N K 1=P A R K 6$ & 1p35-36 & AR & Early & Slow progression & - \\
\hline PARK9 & 1p36 & AR & Juvenile & $\begin{array}{l}\text { Spasticity } \\
\text { Dementia } \\
\text { Supranuclear ophthalmoparesis }\end{array}$ & - \\
\hline$\alpha$-synuclein $=P A R K 1$ & $4 q 421$ & $\mathrm{AD}$ & Late & $\begin{array}{l}\text { Lower prevalence tremor } \\
\text { More rapid progression }\end{array}$ & Yes \\
\hline$U C H-L 1=P A R K 5$ & $4 p 14$ & $\mathrm{AD}$ & Late & None & - \\
\hline NR4A2 & $2 q 22-23$ & $\mathrm{AD}$ & Late & None & - \\
\hline PARK3 & 2p13 & $\mathrm{AD}$ & Late & Dementia in some patients & Yes \\
\hline $\begin{array}{l}\text { Triplication of } \\
\alpha \text {-synuclein }=\text { PARK } 4\end{array}$ & 4p14-16.3 & $\mathrm{AD}$ & Late & $\begin{array}{l}\text { Postural tremor in some relatives } \\
\text { Autonomic dysfunction } \\
\text { Dementia } \\
\text { Weight loss early in disease }\end{array}$ & Yes \\
\hline PARK8 & 12p11.2-q13 & $\mathrm{AD}$ & Late & None & No \\
\hline PARK10 & $1 \mathrm{p} 32$ & & Late & None & - \\
\hline
\end{tabular}

AR: Autosomal recessive; AD: Autosomal dominant

Recently, Singleton et al [42] identified $\alpha$-synuclein locus triplication as a cause to PD in a large family (originally designed as PARK 4 mapped to 4p14163) with autosomal dominant $\mathrm{PD}$, ranging clinically from dementia with Lewy bodies to typical PD. The frequency of the $\alpha-$ synuclein locus triplication in general population of familial PD is unknown.

\section{Parkin gene}

The parkin gene is a large gene consisting of over 1.5 $\mathrm{Mb}$ and about 12 exons, which was mapped to chromosome 6q25.2-27 [43]. A mutation in this gene (homozygous exon depletion) was first identified as an autosomal recessive early-onset trait in a Japanese family [43, 44].

Until now, more than 70 mutations on this gene have been associated with the early-onset form of parkinsonism. These mutations may account for PD in as many as 50\% of familial cases of autosomal recessive juvenile parkinsonism $[45,46]$. Clinically, the disease usually begins when the patient is in his/her 20s, and is prominently associated with dystonia and diurnal fluctuations, progressing slowly while accompanied by early and severe levodopa-induced dyskinesis, but no dementia. Pathologically, there is severe neuronal loss in the substantia nigra pars compacta and locus ceruleus; however, intriguing Lewy bodies which are ubiquitinated and thought to be part of the degradation process are absent in brains of parkin patients, suggesting that the mechanism of neurodegeneration may differ from other forms of PD.

The parkin protein product comprises 465 amino-acids was identified as an ubiquitin ligase involved in the process of protein degradation pathway [47], indicating that ubiquitin-mediated proteolysis may play an important role in the pathophysiology of idiopathic Parkinson's disease. Being associated with recessively inherited PD, these mutations in Parkin will lead to the loss or deprived function of E3 ubiquitin ligase in the nigra and striatum of the patients, resulting in the abnormal accumulation of its substrate proteins. A specific form of $\alpha$-synuclein has been found to be a substrate of the parkin gene, thereby linking the two through the ubiquitin system [48].

In conclusion, mutations in the parkin gene represent a frequent cause of familial early-onset and isolated juvenile parkinsonism, thus making it one of the best genetic markers of familial early onset PD yet.

\section{UCH-L1 gene}

The ubiquitin carboxy-terminal hydrolase L1 (UCH-L1) gene on chromosome 4 encodes a protein which belongs to the family of deubiquitinating enzymes. These enzymes are involved in the ubiquitin/26S proteasome degradation pathway and are also found to be present in Lewy bodies. Researchers have identified a missense mutation in exon 4 of the gene in 2 siblings from a German family with Ileu to 
Met substitution at position 93 (Ile 93Met) on the UCH-L1 protein. However, mutations in $U C H-L 1$ gene have not been found in any other families. UCH-L1 protein is richly expressed in brain, constituting possibly $1 \%$ of brain protein [49]. Its function is unknown, though it is presumed to act to recycle ubiquitin by hydrolyzing the ubiquitinated peptides, the products of the proteasome. The capability of cleaving ubiquitin carboxyl-terminal amides was damaged to $50 \%$ by mutations in the $U C H-L 1$ gene [50]. It is tempting to speculate that the enzyme plays a role in modifying damaged or aggregated proteins that otherwise might accumulate to toxic levels in the neuron.

\section{PINK1 gene}

The PTEN-induced putative kinase 1 (PINK1) gene contains eight exons spanning $\sim 1.8 \mathrm{~Kb}$ and encodes a 581 amino acid protein. Two homozygous mutations in PINK1 gene associated with PD were identified recently. G309D was found in a Spanish family and W437OPA substitutions were found in two Italian families [39]. Studies show that PINK1 mutations are associated with PARK6, a locus relating with autosomal recessive, early-onset PD on chromosome 1p35-p36 by autozygosity mapping in a large consanguineous family from Sicily [51]. Subsequent identification of two additional consanguineous families provided additional evidence of the relationship with PARK6 [52].

\section{DJ-1 gene}

The $D J-1$ gene encodes a ubiquitous highly conserved protein. It was shown that mutations on $D J-1$ are associated with PARK7, a monogenic form of human parkinsonism. It was first linked to the PARK7 loucs in a genetically isolated population in the southwest of the Netherlands (it was later confirmed in an Italian family) [53]. The Dutch kindred carried a homozygous deletion of DJ-1, and the affected individuals in the Italian family were homozygous for the L166P mutation. Clinical characteristics of the DJ-1 parkinsonism include a slow progression of symptoms with sustained response to levodopa treatment [54]. The function of the DJ-1 protein remains unknown, but evidence suggests its involvement in the oxidative stress response [55]. Also some findings demonstrate that loss of DJ-1 function leads to neurodegeneration. The observation that DJ-1 may be involved in the oxidative stress response links a genetic defect in this pathway to the development of parkinsonism, with possible implications for understanding the pathogenesis of the common forms of PD [56]. Revealing the physiological role of DJ-1 may promote the understanding of the mechanisms of brain neuronal maintenance.

\section{NR4A2 gene}

NR4A2, or NURR1 gene which encodes a member of nuclear receptor superfamily, is absolutely necessary for the differentiation and maintenance of the nigral dopaminergic neurons. One study showed two specific heterozygous mutations (-291Tdel and $-245 \mathrm{~T} \rightarrow \mathrm{G}$ ) were found in European descent $[57,58]$. The phenotypes of patients with mutations in the NR4A2 gene are actually identical to those with late-onset PD lacking atypical features.

\section{Biochemical markers}

Two major biochemical markers that have been very helpful to recognize the onset of PD are: the loss of the dopamine transporter detected by PET imaging and the presence of the $\alpha$-synuclein protein located in the Lewy body lesions, which are characteristic of PD. Several other biochemical markers in blood and CSF hare also been proposed. The validity of these markers in clinic application is currently under investigation.

\section{Loss of DAT}

Dopamine transporter (DAT) mediates uptake of dopamine (DA) into dopaminergic neurons by an electrogenic, $\mathrm{Na}^{+}$- and $\mathrm{Cl}^{-}$-transport-coupled mechanism. DA and the uptake blockers such as cocaine would bind to both the shared and separate domains on the transporter, which is influenced dramatically by the presence of cations. Regulation of the DAT occurs both by chronic occupancy with blocker and by acute effects of $\mathrm{D}_{2}$ DA receptors or second messengers such as diacylglycerol (protein kinase $\mathrm{C}$ ) and arachidonic acid. The DAT is involved in the uptake of toxins generating Parkinson's syndrome.

The localization of DAT provides the best marker for the integrity of the pre-synaptic dopaminergic systems that are most affected in PD. The concentration of DAT begin to diminish when the neuronal depopulation is over than $50 \%$, which also explains the appearance of neurological deficit symptoms. This is why the concentration of striatal, preferentially putamen DAT concentration is a high sensitivity parameter for the detection of early phases of PD [59].

Although conventional high-resolution imaging techniques such as MRI and CT are not so beneficial in the diagnosis of PD, they are helpful in the differential identification of some other types of parkinsonisms. Scientists are interested in developing highly sensitive diagnostic techniques for early PD by assessing of DAT concentration in the striatum. PET is a technique which measures the emission of positrons from the brain after a small amount of radioactive isotopes, or tracers, have been injected into the blood stream. Till now PET is considered 


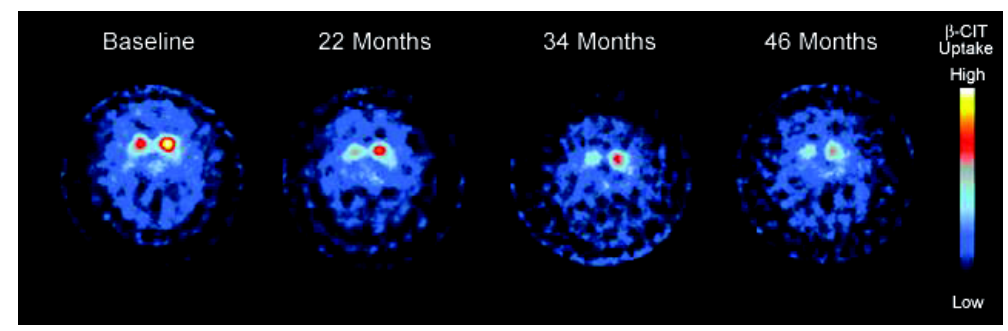

Fig. $2{ }^{123}$ I- $\beta$-CIT SPECT images show progressive decline of nigro-striatal DAT function in patients with Parkinson's disease (PD). Replicated with permission.

to be the most useful tool for PD diagnosis, namely Fl 18 Fluorodopa [60]. Another useful imaging technology for diagnosing PD is single photon emission computed tomography (SPECT), which is similar to PET except for that it uses isotopes with longer half-lives that can be stored on site. Currently, for DAT imaging, there are several available radiopharmaceuticals. For instance, an SPECT image (Fig. 2) of the brains of a patient with PD was taken using the DAT ligand 123I- $\beta$-CIT [61] to show the progressive deterioration of nigro-striatal DA system. As can be observed from the image, reduced DAT signals were detected in the striatum of the patient with PD. Longitudinal studies including early cases of PD are needed for a better understanding of the course of the loss of striatal DAT function in PD.

\section{Lewy bodies}

The Lewy bodies are found to be a characteristic of PD which contains $\alpha$-synuclein. Human $\alpha$-synuclein protein is relatively abundant in brain and predominantly expressed in pre-synaptic nerve terminals. The protein was first implicated in the pathogenesis of a neurodegenerative disease as a novel peptide, which was unrelated to $\alpha$ amyloid peptide. However, it was isolated from purified amyloid of $\mathrm{AD}$ brains and thus named non- $\mathrm{A} \beta$ component of AD amyloid (NAC) [7]. Analysis of its amino-acid sequence revealed that more than half the molecule consists of six imperfect eleven amino-acids repeats, each of which possesses a conserved six-amino-acid core [62]. Two mutations in $\alpha$-synuclein gene were found to be associated with the rare inherited forms of PD, which led to the discovery of Lewy bodies and Lewy neurites, the characteristic lesions in brains of patients with PD and dementia with Lewy bodies [63]. Both mutations of result in amino acid substitutions within this repeated region. However, it still remains unclear how the mutant encoded proteins assist fibril formation, thus leading the way to the discovery of Lewy bodies. Given that $\alpha$-synuclein is also found in other synucleinopathies such as dementia with Lewy bodies
(DLB) and multiple system atrophy, it should be used in the aid of other diagnostic methods to increase the specificity and sensitivity for PD [64].

\section{Biochemical markers in blood and CSF}

An alternative hematogenous biomarker for PD may arise from studies in the periphery. Several potential markers of oxidative stress such as malondialdehade, superoxide radicals, the coenzyme Q10 redox ratio, and 8hydroxyguanosine from RNA oxidation have been measured in blood and the levels of these markers tend to be abnormally higher in PD compared with control groups [65]. DAT immunoreactivity in lymphocytes is decreased and MAO-B activity in platelet is increased [65]. Elevated plasma homocystein level, a risk factor associated with cardiovascular disease and AD, is also detected in PD [66]. The CSF levels of 8-hydroxy-2'-deoxyguanosine and 8hydroxyguanosine are moderately higher while $\beta$ phenylethylamine is lower in PD [65]. CSF specimens from PD patients, regardless of therapy, contain factors that cause specific dopaminergic (DAergic) cell injury in in vitro studies [67]. It has been demonstrated proinflammatory factor tumor necrosis factor (TNF- $\alpha$ ) in CSF is 3-4 fold higher in PD vs normal and disease controls [67]. These findings provide valuable insight into the nature of the pathogenesis of the disease, but none of them are sufficient to be used as a diagnostic biomarker for PD in clinical practice.

\section{OTHER DISEASES}

Currently, $\mathrm{PD}$ and $\mathrm{AD}$ are the two main neurodegenerative disorders in which significant amounts of knowledge to gain better understand the nature of these disorders have been acquired through the application of the respective biomarkers. However, there are still a few other neurodegenerative diseases in which biomarkers are not yet fully understood, in spite of the fact that some progress has been made. In this section of other Diseases, biomarkers of ALS and HD will be examined. 


\section{Amyotrophic Lateral Sclerosis (ALS)}

ALS is an age-dependent motorneuron neurodegenerative disease characterized by the neuronal death of the upper and lower motor neurons, skeletal muscle atrophy, paralysis, and death. The primary goal for scientists with regards to the biomarkers of ALS is to show direct evidence of motor neuronal degeneration within the brain or spinal cord. Approximately $2 \%$ of all ALS and $20 \%$ of familial cases are associated with mutations in the gene for copper/zinc superoxide dismutase, SOD1 [68]. Animal models (especially experiments done on rodents) have shown that those transgenic ones for mutant human SOD1 develop progressive skeletal muscle atrophy, paralysis and death, similar to human cases. This gene appears to be the best biological marker presently available for familial ALS [69].

ALS is clinically and genetically heterogeneous, and as genetic advances are made, phenotypic and genotypic classification will improve. For example, there is a specific phenotype associated with the recessive SOD1 mutation, D90A, of predominantly UMN [70]. This mutation remains an enigma and is one of the few with a consistent phenotype. Thus, a genetic molecular diagnosis can be made with some confidence from the clinical presentation.

Two more genetic mutations: ALS2 and NEFH have been found to be associated with ALS, leading to speculation of two more possible genetic markers. However, studies have shown that these two mutations are not common causes of the disorder [71].

The biological and surrogate markers are under evaluation for their roles in the diagnosis of ALS, in measuring disease progression during therapeutic trials in ALS, and in developing potential new therapies for the disease. Currently the primary underlying mechanisms or causes of ALS are unknown, although many biological changes resulting from the disease process have been recognized. The cascade of biochemical changes occurring within the motor system in ALS eventually leads to degeneration of the lower and upper motor neurons (LMNs and UMNs), which is responsible for the clinical symptoms and signs of the disease. Ideally, scientists should employ direct evidence of motor neuronal degeneration as the primary biological marker in studies of ALS, but brain or spinal cord biopsies are so hard to obtain that they are forced to use secondary or surrogate markers [72]. A surrogate marker is basically the substitute for another marker to diseaserelated epi-phenomena that nevertheless can be used in the diagnosis or measurement of progression of ALS, as no true biomarkers have yet been confirmed for the disorder.

If there is a known family history of similar diseases for a patient presenting signs and symptoms suggestive of ALS, genetic techniques should be applied to establish the diagnosis and screen for mutations of known genes, such as the SOD1 gene in familial ALS. The diagnosis of ALS in the early stages is very difficult because there is no clear evidence indicating the involvement of UMN and LMN at multi-level damage. LMN denervation on electromyography can assist in confirming the diagnosis of ALS in a patient with clinical signs that are purely restricted to the UMN. Similarly, MRI evidence of corticospinal tract degeneration or evidence of UMN dysfunction as indicated by $1 \mathrm{H}$-magnetic resonance spectroscopy (1 H-MRS) can be used to demonstrate UMN involvement in a patient with signs restricted to the LMN [73]. Unfortunately, no technique for the detection of UMN is sensitive enough since clinical signs usually antedate the appearance of MR abnormalities. Biomarkers which have been advanced as potential surrogate markers for the diagnosis of ALS include increased levels of glutamate in the CSF and blood [74], oxidative products in the blood $[66,75]$, abnormal splicing variants of mRNA from EAAT2 (GLT1) in the CSF [76], and changes of $1 \mathrm{H}$-MRS in levels of N-acetyl aspartate (NAA) and glutamate [77].

\section{Huntington's Disease (HD)}

$\mathrm{HD}$ is an inherited disease characterized by choreiform movements, psychiatric symptoms, and slowly progressive dementia [78]. In adults, HD most often causes involuntary movements, but rigidity also can be a feature of the disease. HD is inherited as an autosomal dominant disorder with complete penetrance. A HD gene (IT15 gene) on chromosome 4 has been identified with an abnormal protein product (huntingtin) identified in the brain [79]. However, the relationship between this protein and the selective loss of neuronal groups in the CNS remains to be established. The mutation was identified genetically as a trinucleotide repeat expansion of CAG sequences within the coding region of IT15 gene. The normal range of CAG repeats is about 15 to 30 repeats; however, one study showed that the expanded HD alleles in Singaporeans consists 40 to 54 CAG repeats [80]. The number of CAG repeats, as one study showed, may aid in the prediction of age of onset and penetrance of HD [81]. For instance, for a 41-year-old individual who has 42 CAG repeats, it predicts a $91 \%$ chance that the individual will have HD onset by the age of 65 [82]. Onset of the disease may be different but usually comes up in midlife, depending on both sex and age. As the expansion of CAG was documented in almost all the cases, it can be assumed that a gene mutation on the IT15 is the cause of HD and thus it could be considered a genetic marker [79].

Similar to the biomarkers of the other neurodegenerative diseases, biomarkers for HD would facilitate an accurate 
evaluation of the effectiveness of new therapies and improve the safety and efficiency of clinical trials. As the mechanism of pathogenesis in HD is not yet clear, biomarkers will be needed at all stages of the diseases and combinations of detection methods will be required. Biomarkers to detect alterations of energy metabolism and oxidative damage are available but require validation. Imaging technologies offer great promise in identifying biomarkers. These modalities include PET and nuclear magnetic resonance spectroscopy (NMRS) [82, 83]. Animal and human studies of biomarkers should be run in parallel and compared with each other in order to take advantage of advances being made in animal models to understand the mechanisms of disease. Using the MR modalities, patients could be serially imaged over months or years with minimal adverse side effects, potentially revealing a great deal of information about the natural history of the disease.

A distinction must be made between state and trait markers. For HD, the ideal trait marker already exists, i.e., the presence of the gene with a CAG expansion. State markers, in contrast, tell the state of the disease once it has become manifest and these are what scientists want to know more about.

\section{CONCLUSION}

Several of the biomarkers that have been identified in the past decade are under investigation for their potential application in the early diagnosis of respective neurodegenerative disorders along with an enormous scope for further research in the areas of both genetic and biochemical markers. Although genetic markers are only detected in certain populations, finding these markers is critical in order to pinpoint the cause and pathogenesis of the disorders. Biochemical markers may provide more valuable information regarding different diagnosis and therapeutic guidance to specific diseases. But individual biomarkers cannot and should not necessarily be used alone to diagnose disease; each biomarker has its own application for specific diseases or for specific stages of disease. Overall, a combination of several biomarkers is usually needed to enhance the accuracy, specificity and sensitivity. Again we must bear in mind that biomarkers are just a part of the clinical evaluation and should be used together with a thorough clinical work-up for best results.

Thus, the ultimate goal for scientists to achieve in the future is to find better biomarkers precisely representing the respective diseases; to maximize the use of biomarkers to detect specific biological, pathological, and biochemical abnormality for the purpose of improvement in clinical diagnosis and treatment; and to help better understand the mechanisms of these neurodegenerative disorders.

\section{ACKNOWLEDGEMENTS}

The writing of this review paper was supported by a grant from 100-Talent Project (Chines Academy of Sciences, No. 2002298), a research grant from the Health and Sciences Center, Shanghai Institutes for Biological Sciences (No. J0041-10402), and a grant from E-Institutes of Shanghai Municipal Education Commission (Neurogenomic Lab, 2004).

Received, Jul 3, 2004

Revised, Aug 17, 2004

Accepted, Aut 21, 2004

\section{REFERENCES}

1 Chung S, Sonntag KC, Andersson T, Bjorklund LM, et al. Genetic engineering of mouse embryonic stem cells by Nurr1 enhances differentiation and maturation into dopaminergic neurons. Eur J Neurosci 2002; 16:1829-38.

2 Peterson DA. Stem cells in brain plasticity and repair; Curr Opin Pharmacol 2002; 2:34-42.

3 Tanaka T, Wada K, Yamamori H, Tanaka S, Kudo T and Takeda M. Biological markers for Alzheimer disease. Seishin Shinkeigaku Zasshi 2003; 105:387-392.

4 Sáez-Valero J, Small DH. Altered glycosylation of cerebrospinal fluid butyrylcholinesterase in alzheimer's disease. Brain Res 2001; 889:247-50.

5 The Ronad and Nancy Reagan Research Institute of the Alzheimer's association and the National institute on aging working group. Molecular and biochemical markers of Alzheimer's disease. Neurobiol Aging 1998; 19:109-16.

6 Mulder C, Scheltens P, Visser JJ, Van kamp G J and Schutgen RB. Genetic and biochemical markers for Alzheimer's disease: recent developments. Ann Clin Biochem 2000; 37:593-607.

7 El-Agnaf OMA, Irvine GB. Aggregation and neurotoxicity of $\alpha$ synuclein and related peptides. Biochem. Soc Trans 2001; 30: 559-65.

8 Barghorn S, Davies P, Mandelkow E. Tau paired helical filaments from Alzheimer's disease brain and assembled in vitro are based on $\beta$-structure in the core domain. Biochemistry 2004; 43:1694-703.

9 St. George-Hyslop PH, Tanzai RE, Polinsky RJ, et al. The genetic defect causing familial Alzheimer's disease maps on chromosome 21. Science 1987; 235:885-90.

10 Sennvik K, Fastbom J, Blomberg M, et al. Levels of $\alpha$ - and $\beta$ secretase cleaved amyloid precursor protein in the cerebrospinal fluid of Alzheimer's disease patients. Neurosci Lett 2000; 14: 169-72.

11 Hardy J, Selkoe D. The amyloid hypothesis of Alzheimer's disease: progress and problems on the road to therapeutics Science 2002; 297:353-56.

12 Levy E, Carman MD, Fernandez-Madrid IJ, et al. Mutation of the Alzheimer's disease amyloid gene in hereditary cerebral hemorrhage, Dutch type. Science 1990; 248:1124-26.

13 Eckman CB, Mehta ND, Crook R, et al. A new pathogenic mutation in the APP gene (I716V) increases the relative proportion of A beta 42(43). Hum Mol Genet 1997; 6:2087-9.

14 Citron M, Oltersdorf T, Haass C, et al. Mutation of the $\beta$ - 
amyloid precursor protein in familial Alzheimer's disease increases $\beta$-protein production. Nature 1992; 360:672-4

15 Wisniewski T, Ghiso J, Frangione B. Peptides homologous to the amyloid protein of Alzheimer's disease containing a glutamine for glutamic acid substitution have accelerated amyloid fibril formation. Biochem Biophys Res Commun 1991;180:1528.

16 Berezovska O, Ramdya P, Skoch J, et al. Amyloid precursor protein associates with a nicastrin-dependent docking site on the presenilin 1-gamma-secretase complex in cells demonstrated by fluorescence lifetime imaging. J Neurosci 2003; 23:4560-6.

17 Janssen JC, Beck JA, Campbell TA, et al. Early onset familial Alzheimer's disease: Mutation frequency in 31 families. Neurology 2003; 60:235-9.

18 Sato S, Kamino K, Miki T, et al. Splicing mutation of presenilin1 gene for early-onset familial Alzheimer's disease. Hum Mutat 1998; Suppl 1:S91-S94.

19 Tysoe C, Whittaker J, Xuereb J, et al. A presenilin-1 truncating mutation is present in two cases with autopsy-confirmed earlyonset Alzheimer disease. Am J Hum Genet 1998; 62:70-6.

20 Rogaev EI, Sherrington R, Rogaeva EA, et al. Familial Alzheimer's disease in kindreds with missense mutations in a gene on chromosome 1 related to the Alzheimer's disease type 3 gene. Nature 1995; 376:775-8.

21 Binetti G, Signorini S, Squitti R, et al. Atypical dementia associated with a novel presenilin-2 mutation. Ann Neurol 2003; 54: 832-6.

22 Citron M, Westaway D, Xia W, et al. Mutant presenilins of Alzheimer's disease increase production of 42-residue amyloid beta-protein in both transfected cells and transgenic mice. Nat Med 1997; 3: 67-72.

23 Lambert JC, Coyle N, Lendon C. The allelic modulation of apolipoprotein E expression by oestrogen: potential relevance for Alzheimer's disease. Med Genet 2004; 41:104-12.

24 Farrer LA, Cupples LA, Haines JL, et al. Effects of age, sex, and ethnicity on the association between apolipoprotein $\mathrm{E}$ genotype and Alzheimer disease. A meta-analysis. APOE and Alzheimer Disease Meta Analysis Consortium. JAMA 1997; 278:134956.

25 Mayeux R, Saunders AM, Shea S, et al. Utility of the apolipoprotein E genotype in the diagnosis of Alzheimer's disease. Alzheimer's Disease Centers Consortium on Apolipoprotein E and Alzheimer's Disease. N Engl J Med 1998; 338:506-11.

26 Sunderland T, Linker G, Mirza N, et al. Decreased $\beta$-amyloid1-42 and increased tau levels in cerebrospinal fluid of patients with Alzheimer disease. JAMA 2003; 289:2094-103.

27 Agdeppa ED, Kepe V, Liu J, et al. 2-Dialkylamino-6acylmalononitrile substituted naphthalenes (DDNP analogs): novel diagnostic and therapeutic tools in Alzheimer's disease. Mol Imaging Biol 2003; 5: 404-17.

28 Sair HI, Doraiswamy PM, Petrella JR. In vivo amyloid imaging in Alzheimer's disease; Diagnostic Neuroradiology 2004; 46:93104.

29 Andreasen N, Sjogren M and Blennow K. CSF Markers for Alzheimer's Disease: Total Tau, Phospho-tau and A $\beta 42$. World J Biol Psychiatry 2003; 4:147-55.

30 Andreasen N, Minthon L, Davidsson P, et al. Evaluation of CSF-tau and CSF-Abeta42 as diagnostic markers for Alzheimer disease in clinical practice. Arch Neurol 2001; 58:373-79.
31 Sjogren M, Andreasen N and Blennow K. Advances in the detection of Alzheimer's disease-use of cerebrospinal fluid biomarkers. Clin Chim Acta 2003; 332:1-10.

32 Blennow K, Vanmechelen E. CSF markers for pathogenic processes in Alzheimer's disease: diagnostic implications and use in clinical neurochemistry. Brain Res Bulletin 2003; 61:23542.

33 Hesse C, Rosengren L, Andreasen N, et al. Transient increase in total tau but not phospho-tau in human cerebrospinal fluid after acute stroke. Neurosci Lett 2001; 297(3):187-90.

34 Kim DK, Seo MY, Lim S, et al. Serum melanotransferrin, p97 as a biochemical marker of Alzheimer's disease. Neuropsy chopharmacology 2001; 25:84-90.

35 Ujiie M, Dickstein DL, Jefferies WA p97 as a biomarker for Alzheimer disease. Front Biosci 2002; 7: e42-7.

36 Desrosiers RR, Bertrand Y, Nguyen QT, et al. Expression of melanotransferrin isoforms in human serum: relevance to Alzheimer's disease. Biochem J 2003; 374(Pt 2):463-71.

37 Shoghi-Jadid K, Small GW, Agdeppa ED, et al. Localization of neurofibrillary tangles and beta-amyloid plaques in the brains of living patients with Alzheimer disease. Am J Geriatr Psychiatry 2002;10:24-35.

38 Dekker M C, Bonifati V, van Duijn CM. Parkinson's disease: piecing together a genetic jigsaw. Brain 2003; 126:1722-33.

39 Valente EM, Abou-Sleiman PM, Caputo V, et al. Hereditary eearly-onset Parkinson's disease caused by mutations in PINK1. Science 2004; 304:1158-60

40 Polymeropoulos MH, Higgins JJ, Golbe LI, et al. Mapping of a gene for Parkinson's disease to chromosome 4q21-q23. Science 1996; 274:1197-9.

41 Kruger R, Kuhn W, Muller T, et al. Ala30Pro mutation in the gene encoding alpha-synuclein in Parkinson's disease. Nature Genet 1998; 18:106-8.

42 Singleton AB, Farrer M, johnson J, et al. a-Synuclein locus triplication causes Parkinson disease. Science 2003; 302:841.

43 Kitada T, Asakawa S, Hattori N, et al. Mutations in the parkin gene cause autosomal recessive juvenile parkinsonism. Nature 1998;392:605-8.

44 Abbas N, Lucking CB, Ricard S, et al. A wide variety of mutations in the parkin gene are responsible for autosomal recessive parkinsonism in Europe. French Parkinson's Disease Genetics Study Group and the European Consortium on Genetic Susceptibility in Parkinson’s Disease. Hum Mol Genet 1999; 8:56774.

45 Pankratz N, Nichols WC, Uniacke SK, et al. Parkinson Study Group. Significant linkage of Parkinson disease to chromosome 2q36-37. Am J Hum Genet 2003; 72:1053-7.

46 Fujita D, Yokota O, Terada S, et al. An autopsy case of juvenile parkinsonism; Neuropathology 2000; 20: 252.

47 Shimura H, Hattori N, Kubo S, et al. Familial Parkinson disease gene product, parkin, is a ubiquitin-protein ligase. Nature 2000; 25:302-5.

48 Shimura H, Schlossmacher MG, Hattori N, et al. Ubiquitination of a new form of alpha-synuclein by parkin from human brain: implications for Parkinson's disease. Science 2001; 293:263-9.

49 Leroy E, Boyer R, Auburger G, et al. The ubiquitin pathway in Parkinson's disease. Nature 1998; 395:451-2.

50 Saigoh K, Wang YL, Suh JG, et al. Intragenic deletion in the gene encoding ubiquitin carboxy-terminal hydrolase in gad mice. 
Nature Genet 1999; 23:47-51.

51 Valente EM, Bentivoglio AR, Dixon PH, et al. Localization of a novel locus for autosomal recessive early-onset parkinsonism, PARK6, on human chromosome 1p35-p36. Am J Hum Genet 2001; 68: 895-900.

52 Valente EM, Brancati F, Ferraris A, et al. European Consortium on Genetic Susceptibility in Parkinson's Disease. PARK6-linked parkinsonism occurs in several European families. Ann Neurol 2002; 51:14-8

53 van Duijn CM, Dekker MC, Bonifati V, et al. Park7, a novel locus for autosomal recessive early-onset parkinsonism, on chromosome 1p36; Am J Hum Genet 2001; 69: 629-34.

54 Dekker MC, Bonifati V, van Swieten JC, et al. Clinical features and neuroimaging of PARK7-linked parkinsonism. Mov Disord 2003; 18(7):751-7.

55 Neumann M, Muller V, Gorner K, et al. Pathological properties of the Parkinson's disease-associated protein DJ-1 in alphasynucleinopathies and tauopathies: relevance for multiple system atrophy and Pick's disease; Acta Neuropathol (Berl) - online publication: 2004 Feb 26.

56 Wilson MA, Collins JL, Hod Y, et al. The 1.1-A resolution crystal structure of DJ-1, the protein mutated in autosomal recessive early onset Parkinson's disease. Proc Natl Acad Sci U S A. 2003; 100:9256-61.

57 Le WD, Xu P, Jankovic J, et al. Mutations in NR4A2 associated with familial Parkinson disease. Nat Genet 2003; 33: 85-9.

58 Le WD, Appel SH. Mutation genes responsible for Parkinson disease. Curr Opi Pharmacol 2004; 4:79-84.

59 Parkinson study group. A Multicenter assessment of Dopamine Transporter Imaging with Dopascan/Spect in parkinsonism. Neurology 2000; 55:1540-7.

60 Huang WS, Chiang YH, Lin JC, et al. Crossover study of (99m) Tc-TRODAT-1 SPECT and $\left({ }^{18}\right)$ F-FDOPA PET in Parkinson's disease patients; J Nucl Med. 2003; 44: 999-1005.

61 Parkinson Study Group Dopamine transporter brain imaging to assess the effects of pramipexole vs levodopa on Parkinson disease progression. JAMA. 2002; 287:1653-61

62 Davidson WS, Jonas A, Clayton DF and George JM. Stabilization of alpha-synuclein secondary structure upon binding to synthetic membranes. J Biol Chem 1998; 273:9443-9.

63 Duyckaerts C, Hauw JJ. Lewy bodies, a misleading marker for Parkinson's disease? Bull Acad Natl Med 2003; 187(2): 277-92.

64 Jellinger KA. Neuropathological spectrum of synucleinopathies. Mov Disord 2003; Suppl 6: S2-12.

65 Michell AW, Lewis SJG, Foltynie T, and Barker RA. Biomarkers and Parkinson's disease. Brain 2004, 127:1693-05.

66 O’Suilleabhain PE, Sung V, Hernandez C, et al. Elevated plasma homocystein level in patients with Parkinson disease. Arch Neurol 2004; 61:865-68

67 Le WD, Rowe D, Jankovic J, et al. Effects of cerebrospinal fluid from patients with Parkinson disease on dopaminergic cells. Arch Neurol 1999; 56:194-200
68 Hand CK, Khoris J, Salachas F, et al. A novel locus for familial amyotrophic lateral sclerosis, on chromosome 18q. Am J Hum Genet 2002; 70(1):251-6.

69 Robberecht W. Genetics of amyotrophic lateral sclerosis. J Neurol 2000; 247: 2-6.

70 Parton MJ, Broom W, Andersen PM, et al. D90A-SOD1 mediated amyotrophic lateral sclerosis: a single founder for all cases with evidence for a Cis-acting disease modifier in the recessive haplotype. Hum Mutat 2002; 20:473.

71 Hand CK, Devon RS, Gros-Louis F, et al. Mutation screening of the ALS2 gene in sporadic and familial amyotrophic lateral sclerosis. Arch Neurol 2003; 60(12):1768-71.

72 Dib M. Amyotrophic lateral sclerosis: progress and prospects for treatment. Drugs 2003; 63:289-310.

73 Karitzky J, Ludolph AC. Imaging and neurochemical markers for diagnosis and disease progression in ALS. J Neurol Sci 2001; 191: 35-41.

74 Heath PR and Shaw PJ. Update on the glutamatergic neurotransmitter system and the role of excitotoxicity in amyotrophic lateral sclerosis. Muscle Nerve 2002; 26:438-58.

75 Simpson EP, Yen AA, Appel SH. Oxidative Stress: a common denominator in the pathogenesis of amyotrophic lateral sclerosis. Curr Opin Rheumatol. 2003; 15(6):730-6.

76 Munch C, Ebstein M, Seefried U, et al. Alternative splicing of the 5'-sequences of the mouse EAAT2 glutamate transporter and expression in a transgenic model for amyotrophic lateral sclerosis. J Neurochem 2002; 82:594-603.

77 Schuff N, Rooney WD, Miller R, et al. Reanalysis of multislice (1)H MRSI in amyotrophic lateral sclerosis. Magn Reson Med 2001; 45: 513-6.

78 Reddy PH, Williams M, Tagle DA. Recent advances in understanding the pathogenesis of Huntington's disease. Trends Neurosci 1999; 22:248-55.

79 Caramins M, Halliday G, McCusker E, and Trent RJ. Genetically confirmed clinical Huntington's disease with no observable cell loss. J Neurol Neurosurg Psychiatry 2003; 74:968-70.

80 Law HY, Ng IS, Yoon CS, et al. Trinucleotide repeat analysis of Huntington's disease gene in Singapore; Ann Acad Med Singapore. 2001; 30(2):122-7.

81 Langbehn D, Brinkman R, Falush D, et al. On behalf of an International Huntington's Disease Collaborative Group dagger. A new model for prediction of the age of onset and penetrance for Huntington's disease based on CAG length. Clin Genet 2004; 65 (4):267-77.

82 Hsu YY, Du AT, Schuff N, and Weiner MW. Magnetic resonance imaging and magnetic resonance spectroscopy in dementias. J Geriatr Psychiatry Neurol 2001; 14:145-66.

83 Reynolds NC Jr, Hellman RS, Tikofsky RS, et al. Single photon emission computerized tomography (SPECT) in detecting neurodegeneration in Huntington's disease. Nucl Med Commun 2002; 23:13-8. 